European Thyroid Journal
Eur Thyroid J 2017;6:57-64

DOI: 10.1159/000457794
Received: October 18, 2016

Accepted after revision: January 19, 2017

Published online: March 17, 2017

\title{
Lymph Node Metastases in Papillary and Medullary Thyroid Carcinoma Are Independent of Intratumoral Lymphatic Vessel Density
}

\author{
Filipe Pereira $^{a}$ Sofia S. Pereira ${ }^{a, c}$ Marta Mesquita $^{b}$ Tiago Morais ${ }^{a}$ \\ Madalena M. Costa ${ }^{a}$ Pedro Quelhas ${ }^{c, f}$ Carlos Lopes $^{d, e}$ Mariana P. Monteiro ${ }^{a}$ \\ Valeriano Leite ${ }^{g, h}$ \\ ${ }^{a}$ Clinical and Experimental Endocrinology, Multidisciplinary Unit for Biomedical Research (UMIB), ICBAS, University \\ of Porto, Porto, 'b Serviço de Anatomia Patológica, Instituto Português Oncologia de Lisboa, Francisco Gentil, Lisbon, \\ 'Instituto de Investigação e Inovação em Saúde (I3S), Universidade do Porto, 'Institute of Molecular Pathology and \\ Immunology of the University of Porto (IPATIMUP), e Instituto de Ciências Biomédicas Abel Salazar, Universidade \\ do Porto, and f Instituto de Engenharia Biomédica (INEB), Universidade do Porto, Porto, and ${ }^{9}$ Faculdade de Ciências \\ Médicas, Universidade Nova de Lisboa, and ${ }^{\mathrm{h}}$ Serviço de Endocrinologia, Instituto Português de Oncologia de \\ Lisboa, Francisco Gentil, Lisbon, Portugal
}

\section{Keywords}

Thyroid · Papillary carcinoma $\cdot$ Medullary carcinoma . Metastasis · Lymph vessels

\begin{abstract}
Background: Blood and lymph vessel invasion are well-recognized markers of tumor aggressiveness, as these are the routes that lead to metastases. Thyroid tumors, depending on the histological variant, tend to have distinctive biological behaviors and use different vascular routes to metastasize, yet the mechanisms underlying the metastatic process are still poorly understood. Objectives: The aim of this study was to assess how the lymph vessel density (LVD) in different histological types of thyroid tumors, and in their surrounding tissue, correlate with the presence of lymph node metastases (LNM) and tumor pathological features. Methods: Lymph vessels of papillary thyroid carcinomas (PTC), of the classical (CVPTC, $n=50$ ) and follicular variants (FVPTC, $n=$ 18 ), and medullary thyroid carcinomas (MTC, $n=34$ ) were
\end{abstract}

\section{KARGER}

E-Mail karger@karger.com

www.karger.com/etj immunohistochemically stained against antigen D2-40. The stained area was quantified using a computerized morphometric analysis tool and correlated with the tumor pathological characteristics. Results: LVD within all analyzed thyroid tumor subtypes was significantly lower than in the surrounding thyroid tissues $(p<0.001)$. Despite intratumoral LVD being significantly higher in CVPTC than in FVPTC, and peritumoral LVD being significantly higher in MTC than in PTC $(p<0.05)$, no correlations were found between LVD (either intratumoral or peritumoral) and the presence of lymph node metastasis. Conclusions: As no LVD differences were found amongst thyroid tumors with or without LNM, dissemination is more likely to depend on the tumor ability to invade the abundant lymph vessel network of the surrounding thyroid tissue than on the ability of the tumor to promote de novo lymphangiogenesis.

(C) 2017 European Thyroid Association Published by S. Karger AG, Basel

Filipe Pereira and Sofia S. Pereira contributed equally to this work.
Valeriano Leite

Serviço de Endocrinologia Instituto Português de Oncologia de Lisboa, Francisco Gentil R. Prof. Lima Basto, PT-1099-023 Lisbon (Portugal)

E-Mail vleite@ipolisboa.min-saude.pt 


\section{Introduction}

Malignant thyroid tumors provide a good model to study the mechanisms underlying the metastatic process. The most prevalent thyroid tumors are papillary (PTC) and follicular (FTC) carcinomas. Although both originate from follicular cells, they tend to metastasize through different routes [1]. PTC metastasizes preferentially via lymph vessels to the regional lymph nodes (LNs), while FTC metastasizes predominantly via the blood vessels into the lungs and bones [2-5]. Notwithstanding, PTC is not a homogeneous entity and encompasses several histological variants, of which the predominant forms are the classical variant (CVPTC), which often invades the lymphatic system and metastasizes to the LNs, and the follicular variant (FVPTC), which resembles FTC pathological behavior by spreading via blood vessels and invading distant organs, such as the lungs, and bones. FVPTC is also more likely to harbor RAS mutations and PAX8/PPAR $\gamma$ rearrangements, molecular alterations usually found in FTC, but not in CVPTC, in which BRAF mutations and RET/PTC translocations typically predominate $[6,7]$. The third most prevalent thyroid tumor is the parafollicular cell-derived medullary thyroid carcinoma (MTC) that, depending on the molecular profile, can also be prone to the development of LN metastasis [8-10]. MTC harboring RET mutations in exons 15 and 16 often metastasize into LNs, while RET mutations in other exons are less likely to present with lymph node metastasis (LNM) [9]. RAS-mutated MTCs, which can occur in $25 \%$ of the tumors, display an intermediate LNMs risk when compared to the previous 2 groups [9].

Although the lymphatic system is an important route for tumor dissemination, the commonly held belief has been that metastasizing cancer cells gain access to this system by invading tumor surrounding vessels because no lymphatic vessels can be demonstrated within the tumor using tracers that require transport through the lymphatic system [11]. Although, this could just reflect the collapse of lymphatics due to the high interstitial pressure within the tumor [12], de novo intratumoral lymphangiogenesis has not been considered as a necessary condition for the metastatic process. However, with the recent identification of suitable markers, such as LYVE-1 and D2-40, with which we can distinguish blood from lymphatic vascular endothelium, it has been possible to analyze tumor lymphatics regardless of their ability to transport fluid, and convincing evidence for tumor lymphangiogenesis has begun to accumulate [13-15].

In thyroid cancer, few studies have addressed the relationship between lymphatic vessel density (LVD) and re- gional LN spread $[8,11-13]$. In PTC, the presence of LN metastases has been found to correlate with intratumoral but not with peritumoral LVD [11]. Except for Eloy et al. [13], all studies were hampered by the fact that PTCs with different metastization patterns, such as the classical and follicular variants, have not been considered separately in the analysis. Hence, it is necessary to take into consideration these 2 histological subtypes, which have different clinical, morphological, and molecular features, to reliably correlate the intratumoral or peritumoral LVD with the presence of LNM. Furthermore, the presence of LNM has only been defined at the time of thyroidectomy and a long-term follow-up has not been required in order to classify the nodal status of the patients. In MTC, LVD analysis has also seldom been performed and no attempts to assess their correlation with LNMs have been made [8, $10]$.

On top of these limitations, LVD has been assessed by the hot spot analysis method which is highly operator dependent and prone to bias [14]. A more convenient method is computerized morphometric quantification that removes most of the observer subjectivity and allows analysis of the entire tissue area.

In order to gain further insight into the putative mechanisms leading to the differential dissemination pathways used by thyroid tumors, we examined LVD and its correlation with patient clinical and tumor pathological features in CVPTC, FVPTC, and MTC.

\section{Materials and Methods}

\section{Patients and Tumors}

Paraffin-embedded thyroid tissues $(n=102)$ obtained after thyroidectomy for thyroid tumors from patients under routine follow-up at the Portuguese Institute of Oncology in Lisbon were used. These included CVPTC $(n=50)$, FVPTC $(n=18)$, and MTC $(n=34)$. All patients had been submitted to total thyroidectomy, with central neck dissection for patients with MTC and PTC who had preoperative or intraoperative detection of neck metastases. Prophylactic lateral dissection was performed in MTC with central metastases. Therapeutic lateral dissection was performed in PTC and MTC with lateral positive LNs. Patients had a median followup time of 12 years (range: 4-23) and the lymph node status was determined at the time of the last follow-up appointment (Table 1). The project was approved by the review board of our institution. Informed consent was waived given the retrospective nature of the study.

\section{D2-40 Immunohistochemistry}

Immunohistochemistry was performed in $3-\mu \mathrm{m}$ formalinfixed paraffin embedded tissue sections mounted on adhesive microscope slides (StarFrost, Knittel Glass, Germany). Sections were deparaffinized, rehydrated in graded alcohols, and underwent an-
58

Eur Thyroid J 2017;6:57-64

DOI: $10.1159 / 000457794$
Pereira/Pereira/Mesquita/Morais/Costa/

Quelhas/Lopes/Monteiro/Leite 
Table 1. Patient demographics and tumor pathological features

\begin{tabular}{llll}
\hline & CVPTC $(n=50)$ & FVPTC $(n=18)$ & MTC $(n=34)$ \\
\hline Sex, M:F & $7: 43$ & $7: 11$ & $14: 20$ \\
Age, years & $40(14-82)$ & $45(9-74)$ & $59(31-83)$ \\
Largest tumor diameter, mm & $19.16 \pm 1.55(6-70)$ & $30.67 \pm 3.82(8-60)$ & $34.06 \pm 4.45(8-120)$ \\
T (T1:T2:T3:T4) & $23: 13: 11: 3(46: 26: 22: 6)$ & $3: 7: 8: 0(17: 39: 44: 0)$ & $11: 10: 6: 7(32: 29: 18: 21)$ \\
Extrathyroid extension & $19(38)$ & $3(17)$ & $10(29)$ \\
Lymph node metastases & $25(50)$ & $4(22)$ & $22(65)$ \\
Distant metastasis & $4(8)$ & $2(11)$ & $6(18)$ \\
Thyroiditis & $12(24)$ & $4(22)$ & $3(9)$ \\
\hline
\end{tabular}

Values are presented as $n(\%)$, medians (range), or means \pm SEM (range). CVPTC, classical variant of papillary thyroid carcinoma; FVPTC, follicular variant of papillary thyroid carcinoma; MTC, medullary thyroid carcinoma.

tigen retrieval performed by microwave treatment in $0.01-\mathrm{M} \mathrm{ci-}$ trate buffer at $\mathrm{pH}$ 6.0, during $20 \mathrm{~min}$. Endogenous peroxidase inhibition was performed using hydrogen peroxide (MERK, Germany) at $0.3 \%$, for $15 \mathrm{~min}$, before incubation with the primary antibody for D2-40 (rabbit, 1:200, ref.: S0809, Dako, USA) for $1 \mathrm{~h}$ at room temperature. The detection of the immune reaction was performed using the commercial Dako REAL ${ }^{\mathrm{TM}}$ EnVision ${ }^{\mathrm{TM}}$ Detection System (ref.: K5007), by incubation during $60 \mathrm{~min}$, which includes a dextran backbone with peroxidase (HRP) molecules coupled to goat secondary antibody molecules against rabbit immunoglobulins. DAB (3, $3^{\prime}$ - diaminobenzidine), also included in the same commercial Dako Kit, was used as chromogen and Mayer's hematoxylin at 1:10 dilution in water (ref.: HX390929, Germany) was used for nuclear counterstaining. Normal thyroid tissue was used as the positive control and the omission of primary antibody incubation was used as the negative control.

\section{Computerized Image Analysis}

Hematoxylin-eosin (HE)-stained slides were used for tumor area delimitation based on morphometric criteria by the same experienced pathologist with no access to patients' clinical information. This area delimitation was then transferred to the IHQstained slides before scanning using the image acquisition Olympus VS110 virtual slide scanning system, and captured with a magnification of $20 \times$ using the image acquisition software VSASW (Version 2.3 for Windows).

After manual verification of the tissue images and elimination of artefacts, such as black ink staining, lymphoid aggregates in thyroiditis foci, and tissue overlaps, images were analyzed using the image-processing software FIJI (Life Line Version from June 2, 2014 for Macintosh, National Institutes of Health, USA) after 75\% resizing using the cellSens Dimension software (Version 1.11 for Windows, Olympus).

Using the ROI Manager Tool of FIJI, 3 peritumoral perimeters were delimitated: the first at a distance of 250 pixels from the tumor limit (offset 1), the second at a distance of 250 pixels from offset 1 (offset 2), and the third at a distance of 250 pixels from offset 2 (offset 3). A total peritumoral area (total offset) was obtained through the sum of the 3 peritumoral areas delimited by the 3 offsets. Using FIJI color deconvolution plugin (H Dab), which allows the separation of the stained area from the initial image,

Lymph Vessel Density in Thyroid Tumors based in the RGB system, the stained area with the D2-40 antibody in the total tissue areas of the tumor, each offset, and normal thyroid tissue was quantified as previously described [14]. The percentage of the stained area for the different analyzed regions was assessed by calculating the ratio between the D2-40 stained area in these regions and the respective total area.

\section{Statistical Analysis}

The continuous variables are represented as means \pm standard error of the mean (SEM). For continuous variables that passed the normality test using the D'Agostino and Pearson test, a 1-way ANOVA test with the post-hoc Tukey was used to compare the means of 3 or more groups, and the Kruskal-Wallis test with a post-hoc Dunn test was used for the variables that did not pass the normality test. The correlations between continuous variables were evaluated using the Spearman test. A $\chi^{2}$ test was used to compare the nominal variables between the groups.

Statistical analysis was performed using the GraphPad Prism (version 6.00 for Windows) and the SPSS software (version 22.00 for Windows). $p<0.05$ was considered statistically significant.

\section{Results}

\section{Tumor, Peritumoral, and Normal Thyroid Tissue Lymph Vessel Density}

Immunohistochemistry targeting the D2-40 antigen, an O-linked sialoglycoprotein found on the lymphatic endothelium, allowed lymph vessels staining in the thyroid tumors and surrounding tissues. As documented by previous studies, we observed that in thyroid tumors, lymphatic vessels were either absent, particularly in the center of the tumor, or when present were very small, irregular, and with an ill-defined lumina $[11,13]$. This contrasts with the abundant network of well-defined and large vessels that were present in the peritumoral or tumor-free surrounding normal thyroid tissue areas

Eur Thyroid J 2017;6:57-64 DOI: $10.1159 / 000457794$ 

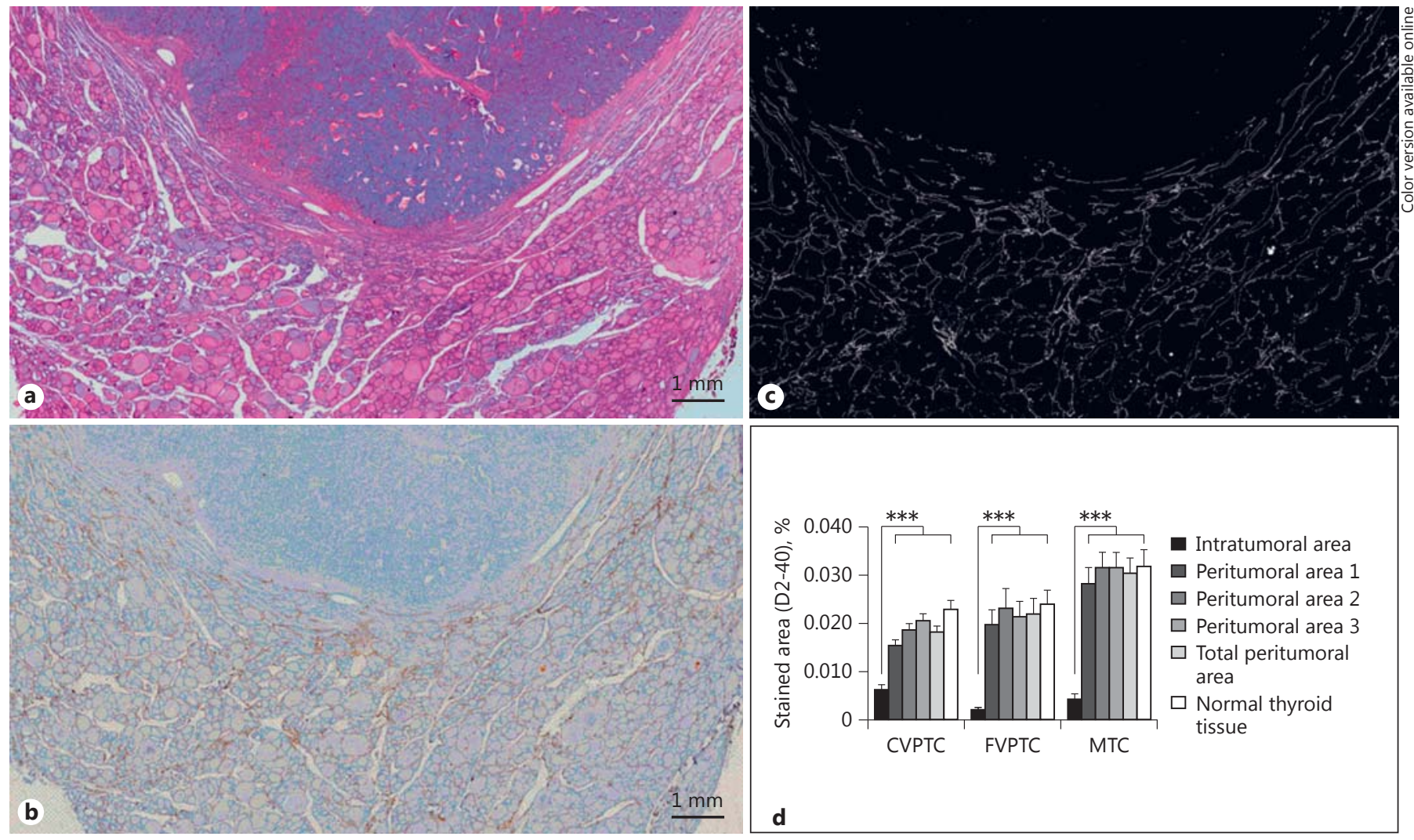

d

Fig. 1. a HE-stained slide of a medullary thyroid carcinoma used for tumor delimitation. b Immunohistochemical staining targeting the D2-40 antigen found on the lymphatic endothelium showed that lymph vessels in thyroid tumors are particularly absent in the center of the tumor, or are very small, irregular, and have an ill-defined lumina when present. c This contrasts with the abundant network of well-defined and large vessels in the peritumoral or tumor-free surrounding normal thyroid tissue areas, highlighted in the negative picture. d Graphic illustration depicting the percentage of anti-D2-40 stained area in the tumor; peritumoral areas 1,2, and 3; total peritumoral area; and normal adjacent thyroid tissue of CVPTC, FVPTC, and MTC. LVD was significantly lower in the tumors when compared with the peritumoral regions or surrounding normal thyroid tissue for any given histological subtype (ANOVA: ${ }^{* * *} p<0.001$ ).
(Fig. 1a-c). LVD was significantly lower within the tumors than in the peritumoral area or surrounding normal thyroid tissue in all the analyzed thyroid tumor histological subtypes $(p<0.001)$ (Fig. 1d). Intratumoral LVD was significantly higher in CVPTC than in FVPTC $(p<0.05)$, and LVD in the peritumoral area $(p<0.001)$ and in the surrounding normal thyroid tissue $(p<0.05)$ was significantly higher in MTC than in PTC (Fig. 2).

\section{Lymph Vessel Density Correlation with Lymph Node}

Invasion, Distant Metastases, Extrathyroid Extension,

Tumor Capsule, and Thyroiditis

LVD in the tumor or peritumoral or disease-free thyroid tissue for any of the analyzed histological thyroid tumor subtypes did not correlate with the presence of lymph node metastases, distant metastases, extrathyroid extension, or thyroiditis (Fig. 3 and online suppl. Figures S1-S3, see www.karger.com/doi/10.1159/000457794). However, lymph node invasion was more frequently associated with extrathyroid extension of the tumor (Table 2). Intratumoral lymph vessel density in FVPTC was significantly higher in noncapsulated tumors $(0.0030 \pm$ $0.0005)$ than in the encapsulated form $(0.0011 \pm 0.0003$, $p<0.01$ ) (data not shown).

\section{Discussion}

Different mechanisms can be used by cancer cells to spread within the body, namely direct invasion of surrounding tissues, spread through the blood vascular system and spread through the lymphatic system. Although 


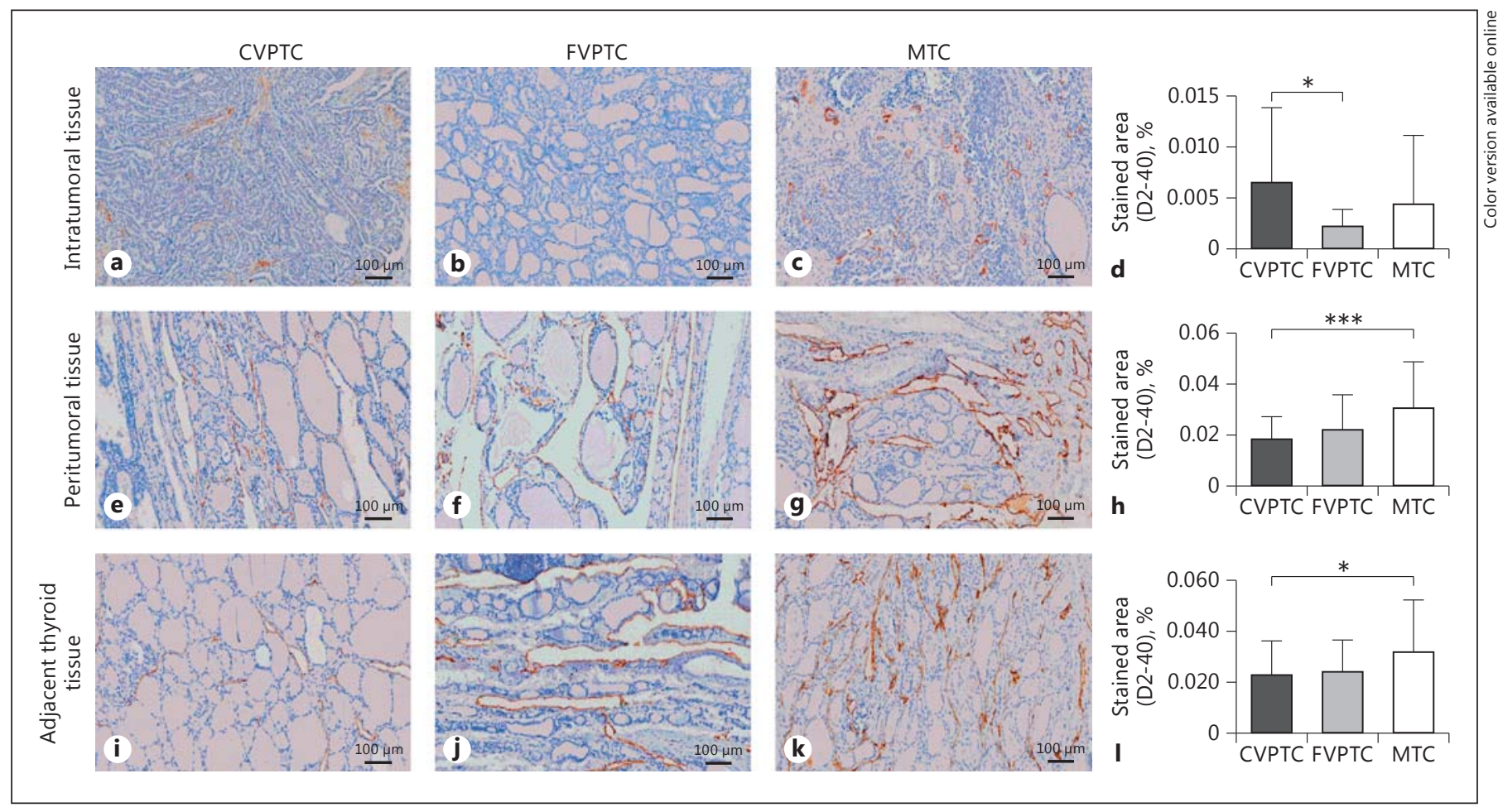

Fig. 2. Microphotographs depicting the immunohistochemical staining for D2-40 of the intratumoral, peritumoral, and surrounding normal thyroid tissue in CVPTC, FVPTC, and PTC. Corresponding graphic illustration showing that intratumoral
LVD was significantly higher in CVPTC than in FVPTC (ANOVA: $p<0.05$ ), while LVD in the peritumoral area (ANOVA: *** $p<0.001$ ) and in the surrounding normal thyroid tissue (ANOVA: $\left.{ }^{*} p<0.05\right)$ was significantly higher in MTC than in PTC.

Table 2. Case distribution according to lymph node invasion and extrathyroid extension

\begin{tabular}{|c|c|c|c|c|c|c|}
\hline & \multicolumn{2}{|l|}{ CVPTC } & \multicolumn{2}{|l|}{ FVPTC } & \multicolumn{2}{|l|}{ MTC } \\
\hline & No & N1 & No & N1 & No & N1 \\
\hline EET absent & $17(56.7)$ & $13(43.3)$ & $13(86.7)$ & $2(13.3)$ & $11(45.8)$ & $13(54.2)$ \\
\hline EET present & $7(36.8)$ & $12(63.2)$ & $1(33.3)$ & $2(66.7)$ & $1(10)$ & $9(90)$ \\
\hline
\end{tabular}

Values are presented as $n$ (\%). CVPTC, classical variant of papillary thyroid carcinoma; FVPTC, follicular variant of papillary thyroid carcinoma; MTC, medullary thyroid carcinoma; EET, extrathyroid extension; N0, absence of lymph node invasion; N1, presence of lymph node invasion.

the lymphatic system is an important route for tumor dissemination, it was a commonly held belief that metastasizing cancer cells gain access to the lymphatic system by invading vessels surrounding the tumor, as within the tumor there are usually no lymphatic vessels [15]. However, the recent identification of suitable markers which allow a clear distinction between blood and lymphatic vessels has demonstrated the presence of lymphatics in tissues and tumors that were previously thought to lack them [16].

Lymph Vessel Density in Thyroid Tumors
The two most frequent carcinomas that originate in the thyroid gland, the PTC and the FTC, although derived from the same follicular cell, tend to metastasize using different vascular pathways. PTC tends to metastasize preferentially via the lymphatic system [11, 17], while FTC usually spreads via the blood system. It has been hypothesized that differences in PTC and FTC dissemination patterns could reflect higher LVD in the papillary carcinomas [17]. Nonetheless, PTC can present with sev-

Eur Thyroid J 2017;6:57-64 DOI: $10.1159 / 000457794$ 


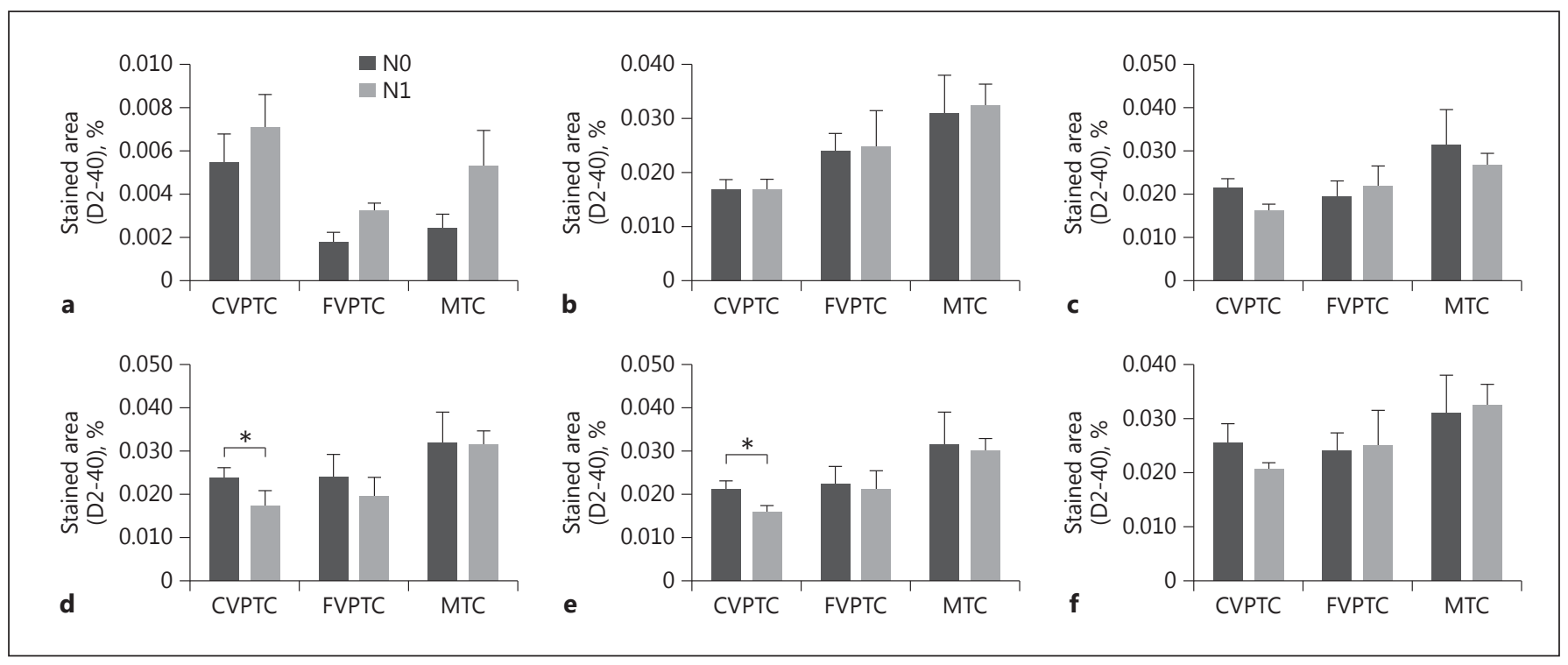

Fig. 3. Anti-D2-40 percentage of stained area in the tumor (a), peritumoral area 1 (b), peritumoral area 2 (c), peritumoral area $3(\mathbf{d})$, total peritumoral area (e), and normal adjacent thyroid tissue (f) in CVPTC, FVPTC, and MTC, according to the presence (N1) or absence (N0) of lymph node invasion. No correlation was found between LVD and presence of LNM.

eral histological variants, with CVPTC and FVPTC being the most common. CVPTC invades preferentially the lymph vessels, whereas in FVPTC the blood vessels are the preferred dissemination route as observed for FTC [18].

Immunohistochemical studies targeting D2-40 in human cancers, including thyroid carcinomas, have confirmed the presence of intratumoral lymphatics. Some of these studies have even detected a correlation between the density of intratumoral lymphatic vessels and the presence of locoregional lymph node metastases $[11,13]$. Hence, these authors have suggested that the number and/or size of lymphatics in a tumor is an important factor for the ability of cancer cells to metastasize to LNs [19], However, thyroid carcinomas are either devoid of intratumoral lymphatic vessels or, when present, such vessels are small, irregular, and have an ill-defined lumina that contrasts with the abundant network of well-defined and large vessels present in the peritumoral tissues areas $[4,15]$, and thus more likely to provide a competent route for lymphatic dissemination. In addition, LVD assessment in thyroid tumors performed in previous studies has several constraints, which include not having had into consideration the different PTC variants when evaluating their correlation with LNM, having determined the lymph node status (N0 or $\mathrm{N} 1$ ) only at the time of thyroidectomy, and having quan- tified LVD using a hot-spot analysis counting grid, which is highly dependent on the selection of a few sample portions of the entire tissue available. Taking into consideration the limitations of the available data, we decided to evaluate LVD in a large number of thyroid tumors using a computerized image analysis tool and to correlate it with the patient clinical and tumor pathological features, after a median follow-up time of 12 years, in order to assess the role of tumor-associated lymphatic density as a tumor progression and dissemination promoting factor.

We, herein, confirm that LVD within thyroid tumors was significantly lower than in the surrounding thyroid tissues. Intratumoral LVD was higher in CVPTC than in FVPTC, and this may potentially be attributed to the higher expression of VEGF-C protein in CVPTC [20]. Still, no difference in LVD was found between N0 and $\mathrm{N} 1$ cases, both for follicular derived and for C-cell derived neoplasms. These results contrast with the findings by Eloy et al. [13] who reported a significant association between intratumoral LVD and the occurrence of lymph node metastases in PTC. Such discrepancy could possibly be attributed to the different methodology used to determine LVD, as the previous study followed the traditional manual process of hotspots analysis that is prone to selection bias whereas in our study, a computerized morphometric analysis method to evalu- 
ate the entire intratumoral and peritumoral tissues was used.

This computerized analysis, despite having the advantage of decreasing the subjectivity of the assessment, still holds the technical constraint related to the loss of the 3 -dimensional nature of the vessels by passing into a 2 -dimensional plane after histological sectioning, as lymph vessels could possibly have been sectioned in various possible plans. Besides that, computerized analysis of any type of tissue presents some challenges, such as the balance between automatic and manual selection of the analyzed area and optimization of the selection of stained area. Before this study, our group used this computerized quantification together with a manual quantification to evaluate the lymphatic vessels density in prostate tumors induced in mice models, and we verified similar results between these two types of methods and found that the computerized analysis is accurate and comparable with more conventional counting methods, but is more expedient [21]. This quantitative method to access the lymphatic vessels density was also used by other groups [22, 23].

In our study, adjoining measures to minimize the variability factors were also undertaken, which included performing the immunohistochemistry technique for all the slide sections on the same day to maintain temperature and humidity conditions constant and undertaking slide analysis by the same observer under similar conditions. For normalizing the image analysis framework, images were acquired on an automated slide scanner using the same brightness parameters, resized to $75 \%$ of its original dimension as the safest percentage reduction to prevent information loss and threshold selection on the FIJI software was duplicated for all images.

Given that lymph node metastases in both PTC and MTC do not correlate with intratumoral LVD, tumor lymphatic dissemination is more likely to depend on the ability of the tumor cells to invade the abundant lymph vessel network of the disease-free thyroid tissue that surrounds the tumor than on the small, irregular, and tiny lymphatic vessels that are present within the tumors, particularly in CVPTC. Encapsulated or partially encapsulated tumors that have an expansive growth pattern, such as FVPTC and probably also FTC, rather than an infiltrative behavior, such as CVPTC and the majority of MTC, are prevented from escaping through the complex network of lymphatic vessels that are present in the surrounding thyroid tissue, which could contribute to explain the differences in the pathologic and clinical behavior observed for the different histological types of thyroid carcinomas.

In conclusion, LVD in thyroid tumors is significantly lower when compared with the surrounding thyroid tissue and no differences in LVD are found amongst thyroid tumors with or without LNM. Therefore, tumor dissemination is more likely to depend on the ability of neoplastic cells to invade the abundant lymph vessel network of the surrounding thyroid tissue than on the ability of the tumor to promote de novo lymphangiogenesis.

\section{Acknowledgement}

The authors would like to acknowledge Dr. Rafael Cabrera for help in tumor selection and Prof. Paula Sampaio, Prof. Eduardo Rocha, Célia Lopes, and Fernanda Malhão for valuable technical support.

The Unit for Multidisciplinary Research in Biomedicine is funded by grants from the Foundation for Science and Technology (UID/Multi/00215/2015). S.S. Pereira holds a PhD grant from the Foundation for Science and Technology (SFRH/BD/89308/2012).

iNOVA4Health - UID/Multi/04462/2013, a program financially supported by Fundação para a Ciência e Tecnologia/Ministério da Educação e Ciência, through national funds and cofunded by FEDER under the PT2020 Partnership Agreement is acknowledged.

\section{Disclosure Statement}

The authors report no conflicts of interest.

\section{References}

1 Xing M: Molecular pathogenesis and mechanisms of thyroid cancer. Nat Rev Cancer 2013;13:184-199.

2 Ito Y, Miyauchi A: Prognostic factors and therapeutic strategies for differentiated carcinomas of the thyroid. Endocr J 2009;56:177192.

3 Vayisoglu Y, Ozcan C: Involvement of level IIb lymph node metastasis and dissection in thyroid cancer. Gland Surg 2013;2:180-185.

Lymph Vessel Density in Thyroid Tumors
4 Lin JD, Chao TC, Hsueh C: Follicular thyroid carcinomas with lung metastases: a 23 -year retrospective study. Endocr J 2004;51:219-225.

5 Muresan MM, Olivier P, Leclere J, Sirveaux F, Brunaud L, Klein M, Zarnegar R, Weryha G: Bone metastases from differentiated thyroid carcinoma. Endocr Relat Cancer 2008; 15:3749.
6 Castro P, Rebocho AP, Soares RJ, Magalhaes J, Roque L, Trovisco V, Vieira de Castro I, Cardoso-de-Oliveira M, Fonseca E, Soares P, Sobrinho-Simoes M: PAX8-PPARgamma rearrangement is frequently detected in the follicular variant of papillary thyroid carcinoma. J Clin Endocrinol Metab 2006;91:213220. 
7 Soares P, Lima J, Preto A, Castro P, Vinagre J, Celestino R, Couto JP, Prazeres H, Eloy C, Maximo V, Sobrinho-Simoes M: Genetic alterations in poorly differentiated and undifferentiated thyroid carcinomas. Curr Genomics 2011;12:609-617.

8 de la Torre NG, Buley I, Wass JA, Turner HE: Angiogenesis and lymphangiogenesis in thyroid proliferative lesions: relationship to type and tumour behaviour. Endocr Relat Cancer 2006;13:931-944.

9 Moura MM, Cavaco BM, Pinto AE, Domingues R, Santos JR, Cid MO, Bugalho MJ, Leite V: Correlation of RET somatic mutations with clinicopathological features in sporadic medullary thyroid carcinomas. Br J Cancer 2009; 100:1777-1783.

10 Tomita T: Tumor-associated lymphatic and venous vessels in medullary thyroid carcinomas. Open J Pathol 2015;5:50.

11 Hall FT, Freeman JL, Asa SL, Jackson DG, Beasley NJ: Intratumoral lymphatics and lymph node metastases in papillary thyroid carcinoma. Arch Otolaryngol Head Neck Surg 2003;129:716-719.
12 Hung CJ, Ginzinger DG, Zarnegar R, Kanauchi H, Wong MG, Kebebew E, Clark OH, Duh QY: Expression of vascular endothelial growth factor-C in benign and malignant thyroid tumors. J Clin Endocrinol Metab 2003; 88:3694-3699.

13 Eloy C, Santos J, Soares P, Sobrinho-Simoes $\mathrm{M}$ : Intratumoural lymph vessel density is related to presence of lymph node metastases and separates encapsulated from infiltrative papillary thyroid carcinoma. Virchows Arch 2011;459:595-605.

14 Pereira SS, Morais T, Costa MM, Monteiro MP, Pignatelli D: The emerging role of the molecular marker p27 in the differential diagnosis of adrenocortical tumors. Endocr Connect 2013;2:137-145.

15 Leu AJ, Berk DA, Lymboussaki A, Alitalo K, Jain RK: Absence of functional lymphatics within a murine sarcoma: a molecular and functional evaluation. Cancer Res 2000;60: 4324-4327.

16 Albrecht I, Christofori G: Molecular mechanisms of lymphangiogenesis in development and cancer. Int J Dev Biol 2011;55:483-494.

17 Giorgadze TA, Baloch ZW, Pasha T, Zhang PJ, Livolsi VA: Lymphatic and blood vessel density in the follicular patterned lesions of thyroid. Mod Pathol 2005;18:1424-1431.
18 Lin HW, Bhattacharyya N: Clinical behavior of follicular variant of papillary thyroid carcinoma: presentation and survival. Laryngoscope 2010;120:712-716.

19 Jackson DG, Prevo R, Clasper S, Banerji S: LYVE-1, the lymphatic system and tumor lymphangiogenesis. Trends Immunol 2001; 22:317-321.

20 Lee SH, Lee SJ, Jin SM, Lee NH, Kim DH, Chae SW, Sohn JH, Kim WS: Relationships between lymph node metastasis and expression of CD31, D2-40, and vascular endothelial growth factors $\mathrm{A}$ and $\mathrm{C}$ in papillary thyroid cancer. Clin Exp Otorhinolaryngol 2012; 5:150-155.

21 Moreira A, Pereira SS, Machado CL, Morais T, Costa M, Monteiro MP: Obesity inhibits lymphangiogenesis in prostate tumors. Int J Clin Exp Pathol 2014;7:348-352.

22 Ozerdem U, Wojcik EM, Duan X, Ersahin C, Barkan GA: Prognostic utility of quantitative image analysis of microvascular density in prostate cancer. Pathol Int 2013;63:277282.

23 Choi J-W, Kim Y, Lee J-H, Kim Y-S: High expression of spindle assembly checkpoint proteins CDC20 and MAD2 is associated with poor prognosis in urothelial bladder cancer. Virchows Arch 2013;463:681-687. 